\title{
Sudden persistent fetal bradycardia after spinal analgesia for labor pain
}

\author{
Yang Hoon Chung, Won Ho Kim, Eun Kyung Lee, and Tae Soo Hahm \\ Department of Anesthesiolgy and Pain Medicine, Samsung Medical Center, Sungkyunkwan University School of Medicine, Seoul, Korea
}

Neuraxial analgesia has become a popular technique for management of labor pain $[1,2]$. However, an increase in the incidence of fetal heart rate (FHR) changes after intrathecal analgesia has been reported $[1,2]$. Although it is known that fetal bradycardia caused by labor analgesia induction does not usually increase the risk of emergent operative deliveries [3], we experienced a case of persistent fetal bradycardia after uneventful spinal analgesia, which led to an emergency cesarean section.

A 39-year-old woman (weight $60.9 \mathrm{~kg}$, height $157.4 \mathrm{~cm}$ ) at $37^{+1}$ week of gestational age was admitted for induction of labor. She was diagnosed with preeclampsia and intrauterine growth retardation. On the first day, dinoprostone slow release pessary (Propess $^{\circledR}$, Bukwang Pharma, Seoul, Korea) was inserted into the vagina for seven hours. After removal of the pessary, intravenous infusion of oxytocin was started. After forty-five minutes, the cervix was fully dilated and the patient complained of severe pain (numerical rating scale, NRS; 8). We decided to perform spinal analgesia without epidural catheter insertion due to shortage of time until delivery. The parturient was laid on her left side with her body bent forwards. Spinal analgesia was performed at the L3-4 level with a $25 \mathrm{G}$ Whitacre needle. A mixture of ropivacaine $3.6 \mathrm{mg}$ and fentanyl $20 \mu \mathrm{g}$ was administered intrathecally. No adverse event occurred during the procedure. One minute after the procedure, the analgesia level reached T12, and NRS score for labor pain decreased from 8 to 3 . However, at about two minutes after spinal analgesia, fetal heart rate decreased to 5070 beats per minute (bpm) (Fig. 1). Maternal blood pressure was 135/70 mmHg when fetal bradycardia developed. Oxytocin infusion was stopped. The parturient was laid in the left lateral de- cubitus position, and intravenous (IV) hydration was provided; however, fetal bradycardia persisted. Tocolytic agents were not used. Fourteen minutes after spinal analgesia, emergency cesarean section was performed by the attending obstetrician.

General anesthesia was induced by IV infusion of thiopental sodium $(5 \mathrm{mg} / \mathrm{kg})$, and succinylcholine $(1 \mathrm{mg} / \mathrm{kg})$. Anesthesia was maintained with $\mathrm{O}_{2}, \mathrm{~N}_{2} \mathrm{O}$ and 1-1.5 vol\% sevoflurane. A male baby with a body weight of $2.03 \mathrm{~kg}$ was delivered. He showed whole body cyanosis and could not establish self respiration at birth with a heart rate below $100 \mathrm{bpm}$. The Apgar

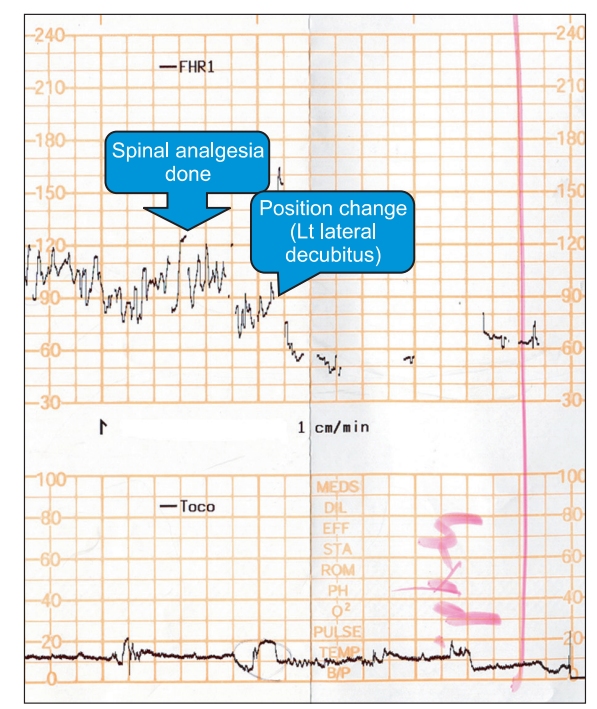

Fig. 1. Fetal heart rate, and the tocogram at the time of fetal bradycardia.

Corresponding author: Tae Soo Hahm, M.D., Department of Anesthesiology and Pain Medicine, Samsung Medical Center, Sungkyunkwan University School of Medicine, 50, Ilwon-dong, Gangnam-gu, Seoul 135-710, Korea. Tel: 82-2-3410-2470, Fax: 82-2-3410-0361, E-mail: tshahm@ skku.edu

(c) This is an open-access article distributed under the terms of the Creative Commons Attribution Non-Commercial License (http:// creativecommons.org/licenses/by-nc/3.0/), which permits unrestricted non-commercial use, distribution, and reproduction in any medium, provided the original work is properly cited. 
score was $1 / 2 / 2$ at $1 / 5 / 10$ minutes after birth, respectively. After endotracheal intubation, the baby was transferred to the neonatal intensive care unit. Brain computed tomography revealed hemorrhagic infarction in the right middle cerebral artery territory along with hematoma in the right occipital lobe. The baby was discharged from the hospital one month later and regular follow-up of rehabilitation was continued.

FHR changes after neuraxial analgesia are not uncommon [1-3]. One possible pathophysiological mechanism is that rapid pain relief causes a short-term imbalance between maternal epinephrine and norepinephrine levels, leading to uterine hyperstimulation [1-3]. But the exact mechanisms are still unknown. The type of neuraxial block could affect the incidence of FHR abnormalities. Abrao et al. [4] reported that combined spinalepidural analgesia (CSE) is associated with a significantly higher incidence of FHR abnormalities than epidural analgesia. They suggested that faster pain relief results in a higher probability of uterine hyperactivity [4]. However, this mechanism has not been clearly demonstrated in a study. Van de Velde et al. [2] demonstrated that spinal analgesia using intrathecal bupivacaine (2.5 mg), sufentanil $(1.5 \mu \mathrm{g})$ and epinephrine $(2.5 \mu \mathrm{g})$ provided a equally fast pain relief as that by spinal analgesia using high-dose intrathecal sufentanil $(7.5 \mu \mathrm{g})$ only. However, FHR changes were more frequent in those who were administered a higher dose of intrathecal sufentanil. Based on this result, it seems that highdose intrathecal opioid plays a role in causing FHR changes, and the onset of rapid analgesia is not the most important causative factor.

In our case, we used $0.2 \%$ ropivacaine $(3.6 \mathrm{mg})$ and fentanyl $(20 \mu \mathrm{g})$ intrathecally without epidural analgesia. Our dose of the intrathecal opioid was not as high as that of intrathecal sufentanil $(7.5 \mu \mathrm{g})$, which was used by Van de Velde et al. in their study. Recently, a case-control study reported that the difference between pre- and post-analgesia pain scores and a higher sensory block height were the risk factors for fetal bradycardia following CSE analgesia for labor pain [1]. These results do not explain the development of fetal bradycardia in our case, because the sensory analgesia level after spinal analgesia had only reached T12.

Fortunately, however, it has been reported that neuraxial analgesia-related FHR changes neither increase the rate of operative deliveries nor affect the maternal and neonatal outcomes [3]. In most cases, FHR changes are temporary and can be managed well with conservative methods such as oxygen supply, use of vasopressors, position change and use of tocolytic agents [3]. But, these conservative methods were ineffective in our case and a cesarean section was inevitable.

As fetal distress and cesarean section are relatively common in the delivery room, other causes of FHR abnormalities should be assessed. Holdcroft and Dob [5] suggested that some iatrogenic events during regional analgesia such as an incorrect infusion dose of oxytocin can precipitate fetal distress. The other possible iatrogenic factors should also be excluded because spinal analgesia could be an innocent bystander in such cases. Cheng et al. [1] reported that the mean time between CSE and fetal bradycardia is 24.1 minutes (SD 16.93). In our case, fetal bradycardia developed after spinal analgesia within a shorter time period ( 2 minutes). This could just be a coincidence. But, there was no evidence of oxytocin overdose and there were no risk factors for fetal bradycardia. It is not clear which factor caused FHR abnormalities in our case.

As mentioned above, there is an increased incidence of fetal bradycardia during labor analgesia due to various reasons. The anesthesiologists should remember that there is a possibility of development of fetal heart rate abnormalities after labor analgesia. Hence, careful fetal heart rate monitoring is necessary after labor analgesia.

\section{References}

1. Cheng SL, Bautista D, Leo S, Sia TH. Factors affecting fetal bradycardia following combined spinal epidural for labor analgesia: a matched case-control study. J Anesth 2013; 27: 169-74.

2. Van de Velde M, Teunkens A, Hanssens M, Vandermeersch E, Verhaeghe J. Intrathecal sufentanil and fetal heart rate abnormalities: a double-blind, double placebo-controlled trial comparing two forms of combined spinal epidural analgesia with epidural analgesia in labor. Anesth Analg 2004; 98: 1153-9.

3. Van de Velde M. Neuraxial analgesia and fetal bradycardia. Curr Opin Anaesthesiol 2005; 18: 253-6.

4. Abrão KC, Francisco RP, Miyadahira S, Cicarelli DD, Zugaib M. Elevation of uterine basal tone and fetal heart rate abnormalities after labor analgesia: a randomized controlled trial. Obstet Gynecol 2009; 113: 41-7.

5. Holdcroft A, Dob D. Regional analgesia for labour and fetal distress: culprit or innocent bystander? Int J Obstet Anesth 2003; 12: 153-5. 